\title{
Methods and interdisciplinary communication in historical climatology
}

\author{
Chantal Camenisch', S. White'2, M. Bauch ${ }^{3}$, Q. Pei ${ }^{4}$ and C. Rohr ${ }^{1}$
}

\section{1st CRIAS workshop, Bern, Switzerland, 1-2 October 2018}

The recently founded PAGES working group Climate Reconstruction and Impacts from the Archives of Societies (CRIAS) held its first workshop on methods and interdisciplinary communication from 1-2 October 2018 in Bern, Switzerland. CRIAS focuses on the methods of historical climatology, a discipline which deals with three different fields:

1. The reconstruction of climate and weather on the basis of archives of society that contain man-made sources such as chronicles, account books or even pictures (Fig. 1 is an example of an illumination in a Bernese medieval chronicle).

2. The impacts of climate and weather on past societies.

3. The history of climate science and perceptions.

After the opening remarks from steering committee member Sam White, the program began with a panel on the state of the field in Historical Climatology, with a focus on Central Europe and China. The presenters Andrea Kiss, Rudolf Brázdil, Xiuqi Fang and Jie Fei gave insight into their research based on the archives of society on drought in medieval Hungary, on the Central European temperature and precipitation series, the unique Chinese historical records, which go back for more than 2000 years, and on the water-level changes of Lake Nansi during the
Qing dynasty. This panel gave participants the possibility to compare the rich historiographic tradition of China with that of parts of Europe, which will be one of the goals of this working group in the next years.

The focus of the second panel was on the narrative sources used in Historical Climatology. Qing Pei gave a comprehensive introduction into weather-sensitive Chinese sources, their context of origin and their content. Chantal Camenisch and Lukas Heinzmann presented results from their recent research, which includes climate impacts on society in Rouen, France, from 1315 to 1715 , and weather conditions and climate impacts recorded in an extended and detailed diary written by a monk in the Einsiedeln, Switzerland, monastery during the Late Maunder Minimum.

The third panel was dedicated to phenological observations in documentary sources and the production of climate indices. Because the source density in Europe in the early 14 th century is considerably less dense compared to later centuries, Martin Bauch and Thomas Labbé proposed new ways of using climate indices for the reconstruction of this climatological key period. Melanie Salvisberg presented an indexbased flood reconstruction of the Gürbe river, Switzerland, and flood impacts in the

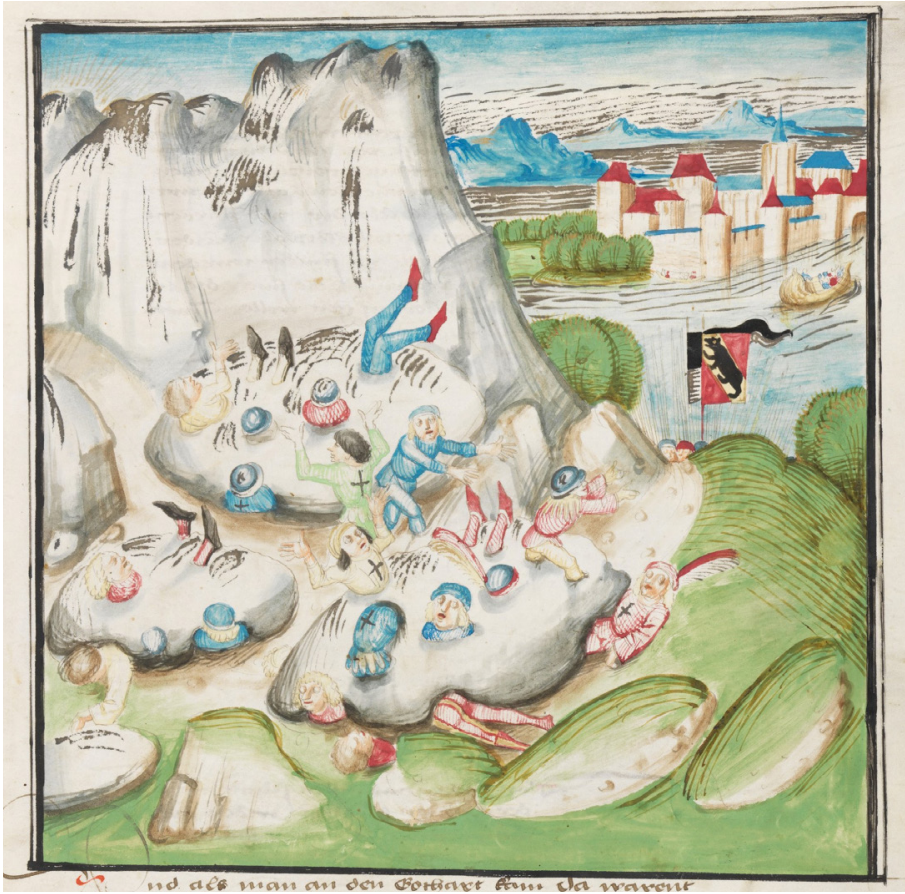

Gürbe valley. Based on the harvest length reported in manorial accounts (account rolls or books in the feudal system), Kathleen Pribyl reconstructed summer precipitation in East Anglia. The last paper of this panel, presented by Christian Pfister and Thomas Labbé, was dedicated to the longest available homogenized grape harvest series from Beaune, France, (1354-2018) and the temperature reconstruction based on that evidence.

New frontiers in Historical Climatology was the topic of the last panel of the workshop. These new frontiers were found in Southern India, where Gemma Ives reconstructed monsoons from 1730 to 1920. Marie-Michèle Ouellet-Bernier presented a sea-ice-cover reconstruction from Nunatsiavut in Labrador which was based on the reports of Moravian missionaries from 1750 to 1950. Dagomar Degroot's presentation focused on the rich information of Dutch ship logbooks. Finally, David Nash talked about precipitation series from South Africa.

Dominik Collet, Rüdiger Glaser, Michael Kahle, Heli Huhtamaa, and Chaochao Gao lead a roundtable focusing on the importance of interdisciplinary collaboration and ways to combine data from the archives of society and data from those of nature. The last part of the workshop comprised discussions in three break-out groups on "preserving, classifying and disseminating data", "climate history as global history", and "comparing Chinese and Central European historical climatology". The aim of the workshop was to determine the future outline of the working group in a broader frame and to bring together Historical Climatologists from different continents. Both aims were achieved in Bern, thanks in part to the financial and administrative support from the Oeschger Centre of Climate Change Research and PAGES.

\section{AFFILIATIONS}

Oeschger Centre for Climate Change Research and Section of Economic, Social and Environmenta History, University of Bern, Switzerland

2Department of History, Ohio State University, Columbus, USA

${ }^{3}$ Leibniz Institute for the History and Culture of Eastern Europe, Leipzig, Germany

${ }^{4}$ Department of Social Sciences, Education University of Hong Kong, China

CONTACT

Chantal Camenisch, chantal.camenisch@hist.unibe.ch

Figure 1: Avalanche killing Bernese and Fribourgese mercenaries at the Gotthard massive in 1478. Bern, Burgerbibliothek, Mss.h.h.I.3, p. 917 - Diebold Schilling, Amtliche Berner Chronik, Bd. 3 (e-codices.ch/de/list/one/bbb/Mss-hh-10003) 